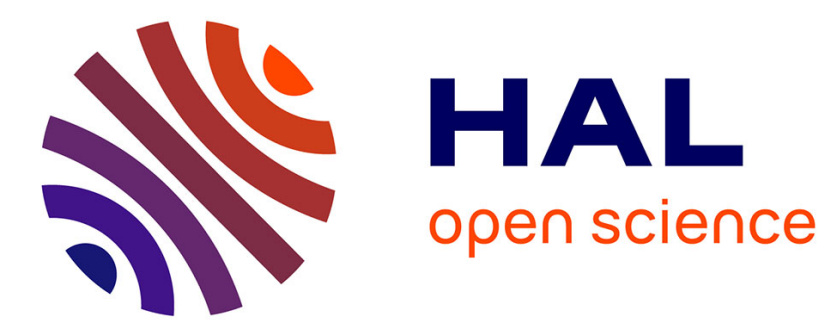

\title{
Conception d'une interface tangible pour l'assemblage en CAO
}

\author{
Ludovic Garreau
}

\section{To cite this version:}

Ludovic Garreau. Conception d'une interface tangible pour l'assemblage en CAO. 17ème Conférence Francophone sur l'Interaction Homme-Machine (IHM'05), Sep 2005, Toulouse, France. pp.339-342, 10.1145/1148550.1148608 . hal-03087676

\section{HAL Id: hal-03087676 https://hal.science/hal-03087676}

Submitted on 24 Dec 2020

HAL is a multi-disciplinary open access archive for the deposit and dissemination of scientific research documents, whether they are published or not. The documents may come from teaching and research institutions in France or abroad, or from public or private research centers.
L'archive ouverte pluridisciplinaire HAL, est destinée au dépôt et à la diffusion de documents scientifiques de niveau recherche, publiés ou non, émanant des établissements d'enseignement et de recherche français ou étrangers, des laboratoires publics ou privés. 


\title{
Conception d'une interface tangible pour l'assemblage en CAO
}

\author{
Ludovic Garreau \\ LIPSI-ESTIA \\ technopole Izarbel \\ 64210, Bidart, France \\ I.garreau@estia.fr
}

\begin{abstract}
RESUME
Dans cet article, nous présentons une interface tangible (TUI), ESKUA, dédiée au domaine de la conception et de la fabrication assistées par ordinateur (CFAO). Notre objectif est de permettre l'anticipation des contraintes d'assemblage du produit dès la phase de conception et notamment lors de la phase de manipulation des modèles géométriques CAO. Pour celà, lors de la phase virtuelle de conception, nous instrumentons la phase d'assemblage du produit avec des objets tangibles afin de matérialiser les contraintes physiques de l'activité d'assemblage.
\end{abstract}

MOTS CLES : Interface tangible, TUI, CAO, suivi vidéo, 3D.

\section{ABSTRACT}

In this paper we present an operational tangible user interface dedicated to the mechanical CAD (Computer Aided Design) parts assembly. This platform is mainly based on the handling of physical objects. In our application field we show that our physical objects lead to take into account assembly constraints from the beginning of the design phase and especially during the phase of CAD model manipulation. We show how the use of our platform leads the designer to have a physical perception of the assembly constraints during the "virtual" phase of design on computer.
\end{abstract}

\section{CATEGORIES AND SUBJECT DESCRIPTORS:}

[User Interfaces] : Prototyping.

\section{GENERAL TERMS: Documentation.}

KEYWORDS: Tangible User Interface, CAD, video tracking, 3D.

\section{INTRODUCTION}

La CAO est une discipline ayant recours aux techniques informatiques pour créer un objet, en achever la forme et générer les données nécessaires à sa fabrication. De nombreux domaines (la mécanique, l'aéronautique, l'architecture) font appel à la conception assistée par ordinateur.

Dans le domaine de l'assemblage mécanique, les logiciels $\mathrm{CAO}$, tout en aidant le concepteur dans sa tâche, masquent des problèmes apparaissant uniquement lors de la phase de fabrication. Par exemple, les difficultés de mise en position relative de deux pièces avant fixation ou encore les difficultés d'insertion d'une pièce par rapport aux autres telles que l'inaccessibilité ou les collisions. En effet, lors de la phase CAO les concepteurs en bureau d'études ne sont jamais confrontés à la réalité du montage.

Notre objectif est de permettre la prise en compte des contraintes réelles le plus tôt possible dans le processus de conception, et de mener ainsi une réflexion accrue sur la phase d'assemblage. Pour cela, nous proposons une alternative aux dispositifs d'interaction classiquement utilisées en CAO avec notre interface tangible (TUI) ESKUA (Expérimentation d'un Système Kinésique Utilisable pour l'Assemblage). Dans cet article, nous présentons les résultats et décrivons le système en donnant l'idée de la démarche.

\section{L'INTERACTION AU MOYEN D'INTERFACES TANGIBLES}

Les techniques d'interaction actuelles ne semblent pas confronter le concepteur aux contraintes réelles de l'assemblage tel la mise en position relative de deux pièces ou le maintien des pièces. La principale raison est l'écart entre les composantes d'action existantes (souris, spaceMouse) et les données virtuelles associées, tant au niveau de la forme, de la couleur et surtout du comportement. Ainsi, un système de Réalité Virtuelle utilisant des gants de données pour sélectionner, manipuler et assembler des objets numériques est capable de rendre le contact entre deux d'entre elles uniquement par une composante de perception visuelle. La composante tactile avec les gants est encore rare. La même application basée sur un phantom [3] ne représente pas exactement les déplacements dans l'espace - à cause d'un déplacement restreint - ainsi la perception liée au comportement est faussée. Aish [1] et Frazer [6] ont developpé une technique d'interaction reposant sur l'idée de proposer des objets réels pour symboliser la donnée : les interfaces tangibles. Les interfaces tangibles (du latin tangere : toucher) utilisent des objets du monde réel pour contrôler et symboliser les données numériques et en permettre une appréhension plus directe. En effet, l'utilisateur a déjà une connaissance de l'objet réel tant au niveau de ce qu'il symbolise que de la façon de l'utiliser. 
Ces objets ont la particularité de rassembler les composants de perceptions visuelles, haptique, du comportement et les composantes d'action. De ce fait, la conception des objets physiques dépend fortement des données numériques manipulées et des actions réalisables. Ullmer et Ishii [7] décrivent et déterminent les caractéristiques des interfaces tangibles. Ainsi, chaque interface tangible est créée en fonction d'un domaine d'application déterminé.

Dans notre cas, le domaine d'application est l'assemblage mécanique et notre interface tangible se nomme ESKUA.

\section{ESKUA}

Notre plateforme tangible ESKUA, se décompose en trois parties, une pour la manipulation, l'autre pour fournir un cadre à la manipulation et la dernière pour récupérer les informations. Dans la suite, nous décrivons successivement les différentes parties d'ESKUA : la partie tangible que nous nommons interacteurs dans cet article, la plateforme et le suivi vidéo.

\section{Partie tangible : les interacteurs}

La partie tangible d'une TUI doit représenter la donnée et son comportement. Les données sont les différentes pièces $\mathrm{CAO}$ et les comportements correspondent à l'assemblage et aux déplacements.

D'un point de vue mécanique, l'assemblage de pièces peut être décrit et simplifié en deux étapes principales : le positionnement respectif des pièces et la fixation mutuelle des pièces. Notre étude de la tâche pour la réalisation des interacteurs s'est principalement appuyée sur les méthodes DFA Design for Assembly [2]. Le principal but des méthodes DFA est d'identifier des critères d'assemblage et de permettre leur anticipation dès la phase de conception afin d'engager des actions préventives au plus tôt. Elles permettent de donner un coefficient d'aptitude à l'assemblage manuel d'un produit en se basant sur l'évaluation d'opérations de base concernant le montage : la préhension, la manipulation et l'insertion de la pièce. Lors de l'utilisation des méthodes DFA, l'utilisateur attribue des volumes simples aux pièces $\mathrm{CAO}$ : cube, cylindre long et court et parallélépipède. Nous avons repris ce principe pour la constitution du premier jeu d'interacteurs détaillé dans [4].

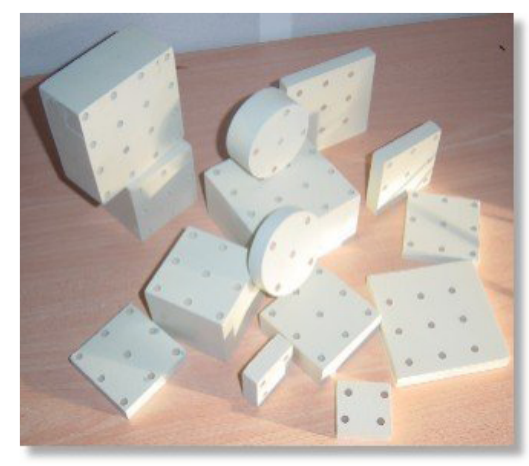

Figure 1. Photographie des interacteurs d'ESKUA.
Notre but était de représenter principalement les assemblages correspondant à des encastrements (assemblages sans mobilités). Ce choix vient du constat que dans la plupart des objets courants, le ratio du nombre de mobilités sur le nombre de pièces est faible. Ware et Rose [8] nous confortent dans notre choix en démontrant que les différences de géométrie et de tailles entre les interacteurs et les pièces $\mathrm{CAO}$ ne sont pas un frein pour la manipulation.

À partir de ce travail de conception, nous avons rédigé un cahier des charges et fabriqué nos interacteurs (figure 1). Dans ce cahier des charges, nous décrivons les contraintes d'utilisation des interacteurs en plus de leurs caractéristiques géométriques. Ils disposent de système de fixation (perçage et accroche par tourillons) pour représenter le comportement d'assemblage. Une étude [5] nous a également amener à ajouter des surfaces fonctionnelles à nos interacteurs. Nous les avons représentées par des petites plaques que nous fixons sur les interacteurs. Actuellement, nous avons douze tailles de cylindres et seize tailles de parallélépipèdes.

\section{L'environnement d'interaction : la plate-forme}

Nous proposons à l'utilisateur un environnement de travail pour fournir un cadre à sa manipulation. Le système conçue (figure 2) ne modifie pas l'espace de travail et peut se ranger et se sortir assez aisément. La plateforme se positionne sous le moniteur dans un caisson. Elle est composée d'un plateau de couleur noire utile à la manipulation et d'un bras télescopique servant à maintenir et positionner la caméra.
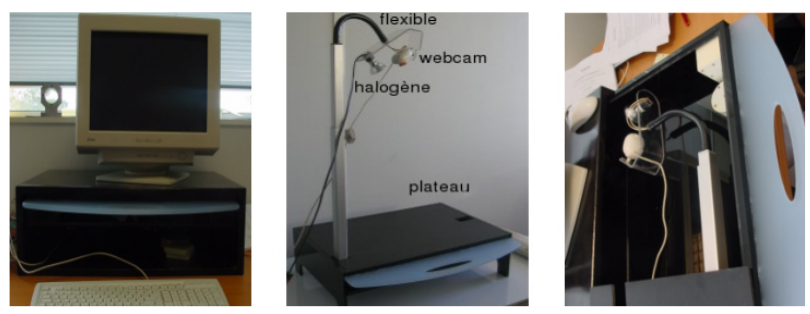

Figure 2. Phographie de la plate-forme d'ESKUA.

La plateforme est principalement constituée de plastique pour des raisons de légèreté et de coût. La caméra est fixée sur un flexible suffisamment rigide pour résister aux contacts et à de légers chocs, mais permettant cependant d'être orienté dans la position souhaitée. Ce flexible est attaché à un bras métallique permettant de le régler à différentes hauteurs.

\section{Le suivi vidéo}

En 2001, au début de ce travail, nous avions délibérément écarté une solution à base de capteurs de positions et d'orientations. En effet, ces capteurs sont encore aujourd'hui trop coûteux, volumineux, et intrusifs car tous munis de fils. Cependant, les capteurs évoluent rapidement ces trois dernières années, leurs tailles diminuent et des solutions sans fils commencent à être existées. Mais ces solutions ne sont pas encore accessibles. En revanche nous pensons que, dés aujourd'hui, le suivi optique offre à l'utilisateur toute la liberté 
dont il a besoin, ne le confronte pas à un système complexe mais au contraire à un système sachant se faire oublié. Il permet également d'envisager une coopération avec d'autres acteurs pour la tâche à effectuer, car les utilisateurs ne sont pas appareillés. Nous proposons un système d'acquisition des données non intrusif basé sur un système de capture et de suivi vidéo avec une caméra.

D'un point de vue matériel, notre système fonctionne avec une webcam Philips Toucam mais également avec une caméra sony XC 55 de meilleure qualitée. Pour la partie logicielle, nous avons envisagé plusieurs solutions (commerciale, de la laboratoire de recherche et personnelle). Dans la version actuelle d'ESKUA, nous utilisons ARtoolkit. ARtoolkit est une bibliothèque conçue pour la réalité augmentée permettant entre autre de détecter les six degrés de liberté d'une cible dans une scène. Ces cibles sont de simples carrés noirs avec des motifs blancs à l'intérieur. Les cibles de type ARtoolkit sont positionnées sur le dessus de nos interacteurs (figure 3 ). Chaque cible est unique et nous permet ainsi d'identifier l'interacteur à tout moment dans l'image. Comme nous identifions chaque interacteur en fonction de sa cible, les occlusions temporaires ne sont pas un problème. D'un point de vue applicatif, la pièce CAO est associée à une cible et d'un point de vue utilisateur la pièce CAO est liée à un interacteur. En revanche, la partie acquisition d'ARtoolkit n'a pas fonctionné pour des problèmes de synchronisation avec la caméra Sony. Nous avons alors modifié au niveau logiciel, le processus classique d'utilisation d'ARtoolkit. Généralement, dans les programme utilisant ARtoolkit, le programmeur utilise $\operatorname{arCapNext()}$ et arVideoGetImage() pour récupérer l'image et utilise ARDetectMarker() pour trouver les cibles. Pour résoudre ce problème de synchronisation, nous avons créé notre propre fonction d'acquisition vidéo pour récupérer l'image et la passer à la la méthode ARDetectMarker(). L'utilisation d'ARtoolkit nous permet de connaître partiellement les six degrés de liberté des interacteurs. Nous utilisons le terme partiellement car nous retrouvons la position de l'interacteur seulement lorsque la cible (positionnée au dessus de celui-ci) est dans le champ de la caméra. Ainsi, nous connaissons la position des interacteurs seulement pour des inclinaisons inférieures à $45^{\circ}$. Au-delà de cette valeur expérimentale, la cible peut ne plus être visible en fonction des conditions de luminosité et de sa position par rapport à la caméra.

La partie suivi vidéo des interacteurs fut compliquée dans notre projet et reste un point à améliorer. L'utilisation d'ARtoolkit, nous a permis de résoudre la plupart des problèmes apparus avec nos algorithmes de suivi vidéo : suivi en temps réel, problèmes après une occlusion, proximité des cibles. Dans cette version, nous connaissons partiellement les six degrés de liberté nos interacteurs. Nous pensons améliorer le suivi en disposant plusieurs cibles sur chaque interacteur. Ainsi, il y aura au moins une cible de chaque interacteur dans le champ de la caméra.

En détectant en permanence une cible appartenant à l'interacteur, ce principe nous permettra de connaître ses six degrés de liberté.

\section{cas d'utilisation}

L'interface graphique est le lien visuel entre les interacteurs et les pièces CAO. Elle est composée (figure 3,1) de trois cadres. Le premier affiche une pré-visualisation $3 \mathrm{D}$ de la pièce CAO sélectionnée. Le second cadre affiche la liste des pièces CAO importées pour la session de travail en cours et permet leur sélection. Le troisième cadre est la vue principale des pièces $\mathrm{CAO}$ associées à des interacteurs. Grâce à l'interface logicielle, l'utilisateur crée un projet et importe les pièces CAO. Pour réaliser l'association entre un interacteur et une pièce $\mathrm{CAO}$, il dispose un interacteur sur le plateau (figure 3, 2a) puis avec la souris, sélectionne une pièce $\mathrm{CAO}$ dans la liste. A partir de ce moment, l'utilisateur manipule l'interacteur pour déplacer la pièce $\mathrm{CAO}$ dans la scène. De la même manière, l'utilisateur ajoute de nouvelles pièces $\mathrm{CAO}$ en utilisant d'autres interacteurs (figure 3, 3a/3b). Toutes les actions de déplacement des interacteurs sont reproduites sur les pièces $\mathrm{CAO}$ associées dans la vue principale (figure 3, $2 b)$. Les positions des interacteurs reflètent les positions des pièces $\mathrm{CAO}$, comme illustrer par les photographies $4 \mathrm{a} / 4 \mathrm{~b}$ et $5 \mathrm{a} / 5 \mathrm{~b}$ de la figure 3 .

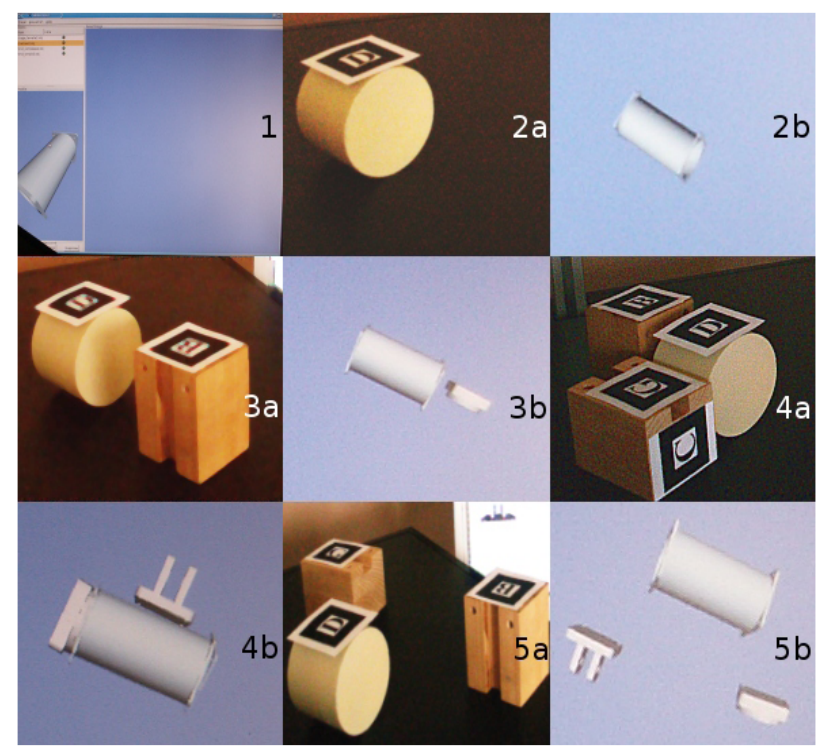

Figure 3. Illustration de l'utilisation d'ESKUA.

\section{CONCLUSION}

Avec ESKUA, l'utilisateur associe intuitivement les interacteurs aux pièces $\mathrm{CAO}$ car le positionnement relatif des interacteurs dans l'espace réel est identique au positionnement des pièces CAO dans l'espace virtuel. En revanche, la différence de taille entre les objets réels et virtuels pose des problèmes pour la tâche d'assemblage. En effet, deux interacteurs en contact n'impliquent pas que les pièces $\mathrm{CAO}$ associées soient en contact et inversement, des pièces $\mathrm{CAO}$ en contact n'impliquent pas que les interacteurs soient en contact. Nous cherchons une solution logicielle à ce 
problème car nous ne pouvons pas reproduire tous les rapports de taille existant entre les pièces $\mathrm{CAO}$ et les interacteurs.

À partir d'ESKUA, un nouveau travail, en collaboration avec l'Institut Français du Pétrole, est en cours pour la conception d'interacteur pour le domaine des géosciences.

\section{REFERENCES}

1. Aish, R. 3D Input for CAAD Systems. Computer-Aided Design, Vol. 11, No. 2, 1979, 66-70.

2. Boothroyd G. and Dewhurst P. Design For Assembly - A Designer's HandBook Boothroyd Dewhurst Inc, Wakerfield, Rhode Island, 1983.

3. Cohen A. and Chen E. Six Degree-of-Freedom Haptic System for Desktop Virtual Prototyping Applications. IN Proceedings of the American Society of Mechanical Engineers Winter Annual Meeting, Dynamics Systems and Control, (November, 1999, Nashville, Etats Unis), 1999, 401-402.

4. Garreau L. and Legardeur J. and Couture N. Tangible Interface for mechanical CAD parts assembly. In Proceedings of Virtual Concept, (November 5-7, 2003, Biarritz, France), 222-227.
5. Legardeur J. and Garreau L. and Couture N.

Experiments to evolve toward a tangible user interface for CAD parts assembly. In Proceedings of Electronic Imaging Science and Technology, Stereoscopic Displays and Virtual Relality Systems XI,(January 16-20, 2004, San Jose, Etats Unis), SPIE, Bellingham, 2004, 438-445.

6. Sutphen S. and Sharlin E. and Watson B.A. and Frazer J. Reviving a tangible interface affording 3D spatial interaction. In Proceedings of eleventh Western Canadian Computer Graphics Symposium, (March 26-29, 2000, Panorama Mountain Village, Canada), $155-166$.

7. Ullmer B. and Ishii H. Tangible Bits : Towards Seamless Interfaces between People, Bits and Atoms. In Proceedings of the Conference on Human Factors in Computing Systems, (April 18-23, 1997, Los Angeles, Etats Unis), ACM Press, New York, 1997, 234-241.

8. Ware C.and Rose J. Rotating Virtual Objects with Real Handles. Association for Computing Machinery Transactions on Computer-Human Interaction, Vol. 6, No. 2, 1999, 162-180. 